\title{
Control of leading edge separation on airfoil using DBD plasma actuator
}

\author{
Nazri MD DAUD*, Yasuaki KOZATO**, Satoshi KIKUCHI*** and Shigeki IMAO*** \\ *Graduate School of Engineering, Gifu University \\ 1-1 Yanagido, Gifu-shi, Gifu 501-1193, Japan \\ E-mail:r3812203@edu.gifu-u.ac.jp \\ **Department of Integrated Mechanical Engineering, Daido University \\ 10-3 Takiharu-cho, Minami-ku, Nagoya 457-8530, Japan \\ ${ }^{* * * D e p a r t m e n t ~ o f ~ M e c h a n i c a l ~ E n g i n e e r i n g, ~ G i f u ~ U n i v e r s i t y ~}$ \\ 1-1 Yanagido, Gifu-shi, Gifu 501-1193, Japan
}

Received 31 January 2014

\begin{abstract}
This study deals with the separation control of an airfoil when a dielectric barrier discharge (DBD) plasma actuator is mounted on its leading edge. The experiments were performed at Reynolds number, $R e \approx 67,000$ in an external airflow of $10 \mathrm{~m} / \mathrm{s}$. The DBD plasma actuator was installed at $x / c=0.025$ of a NACA0015 airfoil with a $100-\mathrm{mm}$ chord and $150-\mathrm{mm}$ width. Flow visualization, lift force and velocity measurements were conducted with on and off modes of the actuator (pulse-modulated drive). We found that the application of pulse modulation condition was able to delay a stall up to angle of attack $\alpha=17^{\circ}$ compared to the Duty $=100 \%$ $\left(\alpha=15^{\circ}\right)$. At $\alpha=17^{\circ}$, the maximum lift coefficient $C_{l}$ was obtained for a non-dimensional pulse modulation frequency $S t$ of 4.0. With an increase in $\alpha$, the lift coefficient $C_{l}$ decreases sharply after reaching a maximum, which is entirely different from the case of $S t=0.6$, where the value of $C_{l}$ falls gradually. For a high angle of attack $\left(\alpha=18^{\circ}\right)$ that exceeds the maximum $C_{l}, S t=0.6$ produces a better lift improvement than when $S t=4.0$. Our results showed that the separation when $S t=4.0$ is larger than that when $S t=0.6$. In addition, we also found that there is an optimum actuation time of about $0.25 \mathrm{~ms}$.
\end{abstract}

Key words : Plasma actuator, Airfoil, Separation control, Dielectric barrier discharge (DBD)

\section{Introduction}

In the past, plasma actuators have been shown to be capable of manipulating airflow by producing an electric wind in the boundary layer. Many researchers have further investigated the potential of this device. As a result, plasma actuators have found many applications such as flow separation control for airfoils (Post and Corke, 2004), flow control around a circular cylinder (Jukes and Choi, 2009), wind turbine performance enhancement (Greenblat, et al., 2012) and the suppression of vortices (Hasebe, et al., 2011).

Recently, many researchers have been studying flow separation control with unsteady actuation mechanisms. For example, Rethmel et al. (2011) investigated flow control for an airfoil with nanosecond-pulse dielectric barrier discharge (DBD) plasma actuators. Asada et al. (2009) also conducted experiments using burst wave plasma actuators in a low-speed wind tunnel. Balcon et al. (2009) studied the airflow reaction on a flat plate with DBD actuation times of between $2 \mathrm{~ms}$ and $400 \mathrm{~ms}$. Amitay and Glezer (2002) focused on the effect of the actuation frequency for manipulating the flow reattachment over a stalled airfoil. It was found that an effective pulse-modulated drive can be achieved even with a $10 \%$ duty cycle (Asada, et al., 2009). This duration (10\% duty cycle) was found to be sufficient to impart momentum to the fluid in the boundary layer and improve the aerodynamic performance.

The effect of unsteady actuation is normally evaluated by the Strouhal number or optimum dimensionless burstwave frequency. However, the effectiveness of the pulse-modulated drive has still not been clearly described for high angle of attack conditions. Different authors have stated findings that vary depending on their methodologies and case studies. For example, Sidorenko et al. (2007) reported optimum dimensionless burst wave frequencies of 2.6 to 14. Rethmel et al. (2011) obtained a value of 2.0. Meanwhile, Asada et al. (2009) and Goksel et al. (2006) obtained 9.1 and 1.0 , respectively. 
Therefore, in this study, we set out to investigate how the flow is affected by a plasma surface discharge when the pulse-modulated drive is applied with a high angle of attack. The DBD plasma actuator was located at the leading edge because this configuration had been found to be more effective at high angles of attack (Jolibois, et al., 2008). The lift coefficient was investigated for each Strouhal number. As a result, we found that effective flow control is possible by using the pulse-modulated drive at high angles of attack.

\section{Nomenclature}

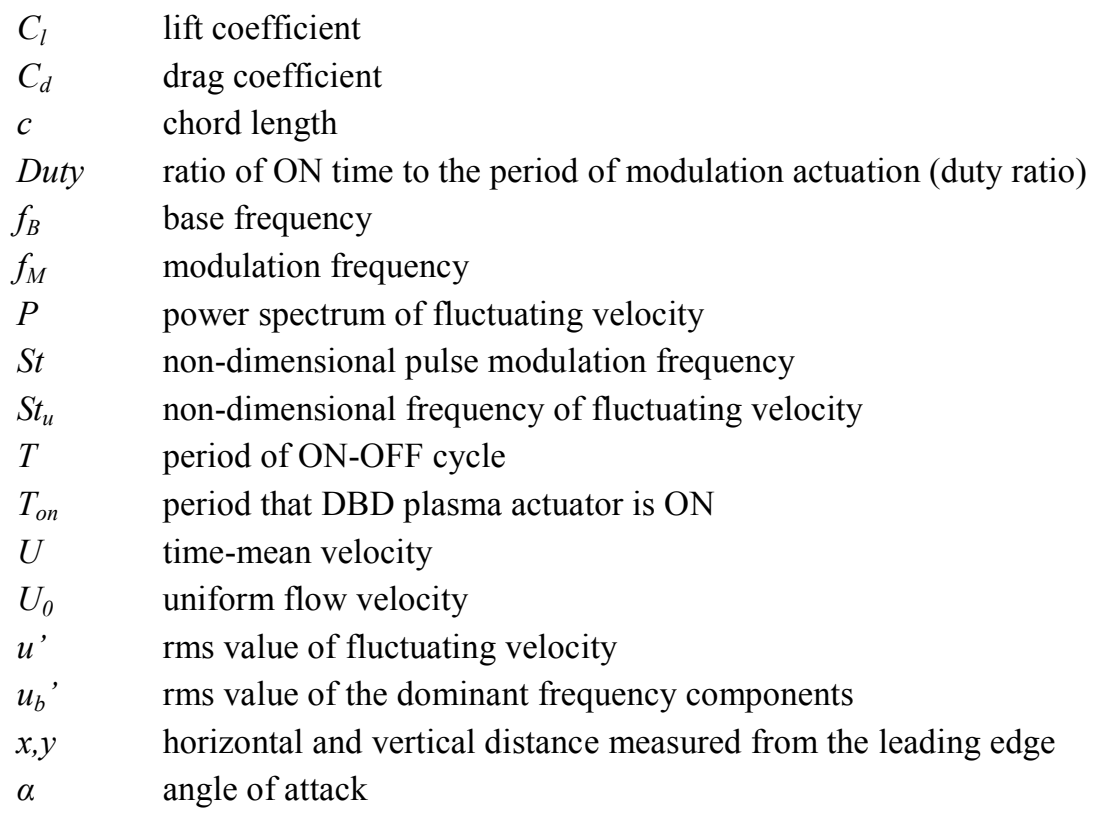

\section{Experimental setup}

Figure 1 shows an overview of the setup used in the experiment. The experiment was conducted in a low-speed, semi-closed wind tunnel with a square cross-section measuring 500 x $500 \mathrm{~mm}$. An NACA0015 airfoil with a chord length of $100 \mathrm{~mm}$ and a span of $150 \mathrm{~mm}$ was tested at $R e \approx 67,000$ in an airflow of $10 \mathrm{~m} / \mathrm{s}$. This Reynolds number was selected to allow us to understand the effect of the plasma actuator on the flow around the airfoil and to clarify the separation control mechanism in flight applications with a low Reynolds number (Sosa and Artana, 2006; Yang, et al., 2007; Greenblatt, et al., 2008). The DBD plasma actuator was installed at $x / c=0.025$ of the chord length. The end plates were made of a clear Plexiglas material to enable the visualization of the flow. We took our measurements at a point at the mid-span of the airfoil. This allowed us to assume that the influence of the end plates, at which boundary layers are formed, was considered to be negligible. This had been proven previously that the three-dimensional (3D) effects did not change the global dynamics of the flow separation (Bernard, et al., 2010). While, we have not applied any correction due to the end plates effect on aerodynamics forces because of the lack of equipment and expertise. Therefore, the measured values of aerodynamic forces were obtained without any compensation. The DBD plasma actuator consisted of two copper-tape electrodes, each 50- $\mu \mathrm{m}$ thick and 5-mm wide. The two electrodes (exposed and encapsulated electrodes) were arranged in parallel with a 1-mm overlap. The electrodes were separated by a $100-\mu \mathrm{m}$ thick Kapton film, which acted as a dielectric.

A high-voltage AC current was supplied to the exposed electrode while the encapsulated electrode was grounded. The base waveform was an $8-\mathrm{kHz}$ sinusoidal wave, generated by a digital function generator (DF1906, NF Corporation, Japan). The signal was amplified by a high-voltage amplifier (HEOPT-10B10-LI, Matsusada Precision Inc, Japan) to give a peak-peak voltage of $6 \mathrm{kV}$. Under these conditions, the DBD plasma actuator produced a maximum velocity of approximately $0.2 \mathrm{~m} / \mathrm{s}$ in quiescent air. Figure 2 shows the time-mean velocity flow field as obtained by Particle Image Velocimetry (PIV) measurement. 
Unsteady actuation was performed by applying a low modulation frequency to the base wave. Figure 3 shows the waveform of the ON-OFF control. A lift force was measured directly by a load cell (LMC-3501-50N, Nissho Electric Works, Japan) with a rated load of $50 \mathrm{~N}$ and an accuracy of $\pm 0.2 \%$. The sampling frequency was set to $1 \mathrm{kHz}$ with a low-pass filtering of $10 \mathrm{~Hz}$ over approximately $4 \mathrm{~s}$. The velocity was measured with an I-type hot-wire probe and a constant-temperature anemometer. The thermal effect caused by the plasma generation was not excessively large as the measurements were not taken in the immediate vicinity of the plasma actuator. The sampling frequency was $50 \mathrm{kHz}$ and the measurement time was approximately $4 \mathrm{~s}$. The measurements were taken at intervals of a minimum of $5 \mathrm{~mm}$ and a maximum of $10 \mathrm{~mm}$ along the $x$-axis, and at a minimum of $1 \mathrm{~mm}$ and a maximum of $5 \mathrm{~mm}$ along the $y$-axis, some distance from the edges of both the exposed and encapsulated electrodes. In addition, electromagnetic noise was removed by using a 2-kHz low-pass filter. The output signal from the anemometer was converted by an A-D converter (WE7000, Yokogawa, Japan), after which the data was processed by a customized LabVIEW program. Flow visualization was conducted by using a high-speed camera (GX-8, nac Image Technology, Japan) and a continuous laser sheet (SVL-532-1000-45L, Sanctity Laser, China). The laser sheet intersected the airfoil at its mid-span, and the axis of the high-speed camera was oriented perpendicular to that of the laser sheet. The camera frame rate was $3000 \mathrm{fps}$ and its resolution was 1024 x 768 pixels. For the experiment, the space inside the wind tunnel was filled with smoke and the airflow speed was set to $5 \mathrm{~m} / \mathrm{s}$. As a result, the Reynolds number for the flow visualization was half that used in our other experiments. However, the trend in the aerodynamic performance did not vary significantly, especially for $S t$ $=0.6$ and $S t=4.0$.
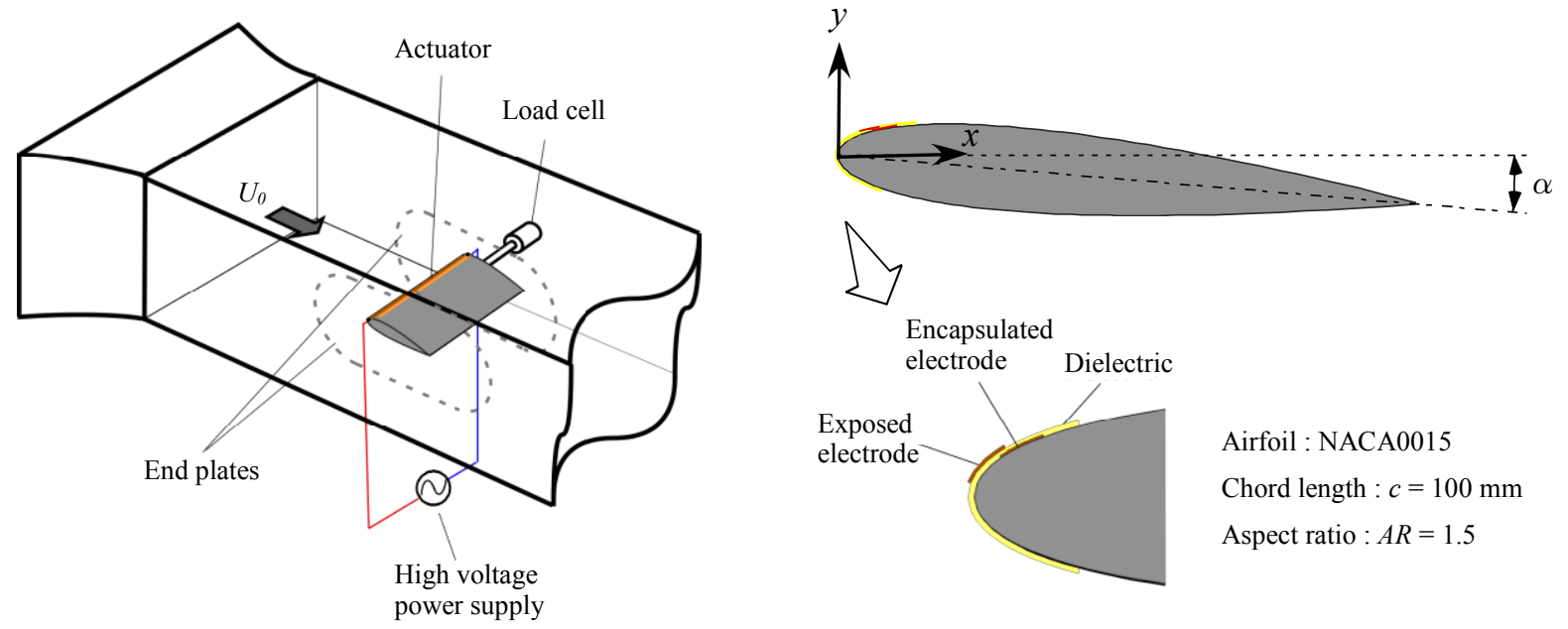

Fig. 1 Experimental setup using airfoil model NACA0015 in a low-speed, semi-closed wind tunnel with $R e \approx 67,000$ and an airflow velocity of $10 \mathrm{~m} / \mathrm{s}$. The DBD plasma actuator consists of encapsulated and exposed electrodes separated by Kapton film. The actuator is located on the airfoil at $x / c=0.025$ of the $100-\mathrm{mm}$ chord length.

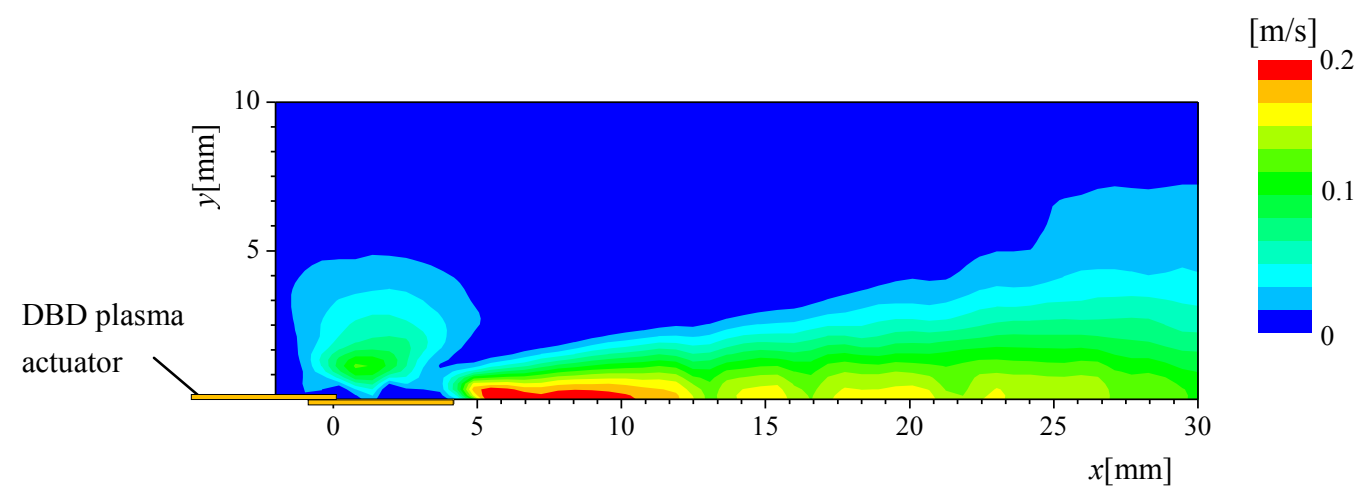

Fig. 2 Contour map of time-mean velocity obtained by PIV measurement when the DBD plasma actuator is applied by Duty $=100 \%$ in quiescent air. The DBD plasma actuator produces a maximum velocity of approximately $0.2 \mathrm{~m} / \mathrm{s}$ between $x=5 \mathrm{~mm}$ and $10 \mathrm{~mm}$. 


\section{Results and discussion}

\subsection{Effect of pulse-modulation drive on airfoil performance characteristics}

This section discusses the effect of the pulse-modulation drive on the lift coefficient. Figure 4 clearly shows that the application of pulse modulation is always able to increase the value of $C_{l}$, relative to a Duty $=100 \%$. At Duty $=$ $100 \%$, the airfoil stalled at $\alpha=15^{\circ}$, but the ON-OFF control maintained the increment of the $C_{l}$ values. In our experiment, the duty ratio was set to $10 \%$ based on the results obtained by Asada et al. (2009). They found that $10 \%$ is the most effective setting for the pulse-modulation drive. From the results of our experiment, we noticed that $S t=0.6$ and $S t=4.0$ exhibited notably different trends (where $S t$ is non-dimensional pulse modulation frequency). At the same time, $S t=0.3$ and $S t=1.0$ exhibited a similar trend to $S t=0.6$, while $S t=2.0$ behaved in much the same way as $S t=$ 4.0. When $S t=4.0, C_{l}$ decreased sharply after reaching a maximum, while $C_{l}$ fell gradually when $S t=0.6$. These findings prompted us to investigate the effect of setting both $S t=0.6$ and 4.0 on the stall control condition $\left(\alpha=16^{\circ}\right)$ and the high angle of attack $\left(\alpha=18^{\circ}\right)$. In this study, the stall control condition is referred to the angle of attack of pulse modulation case when a stall does not occur and for which there is an increase in $C_{l}$ beyond the stall point of the base case.

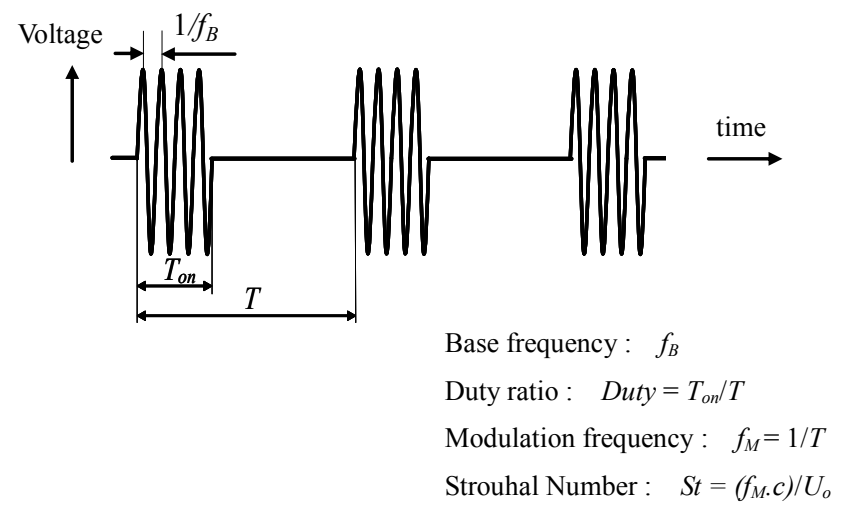

Fig. 3 Typical waveform generated by pulse modulation. The signal is amplified to a peak-peak voltage of $6 \mathrm{kV}$ and a base frequency of $8 \mathrm{kHz}$. In this experiment, $T$ is the period of the ON-OFF cycle and $T_{\text {on }}$ is the period during which the DBD plasma actuator is ON.

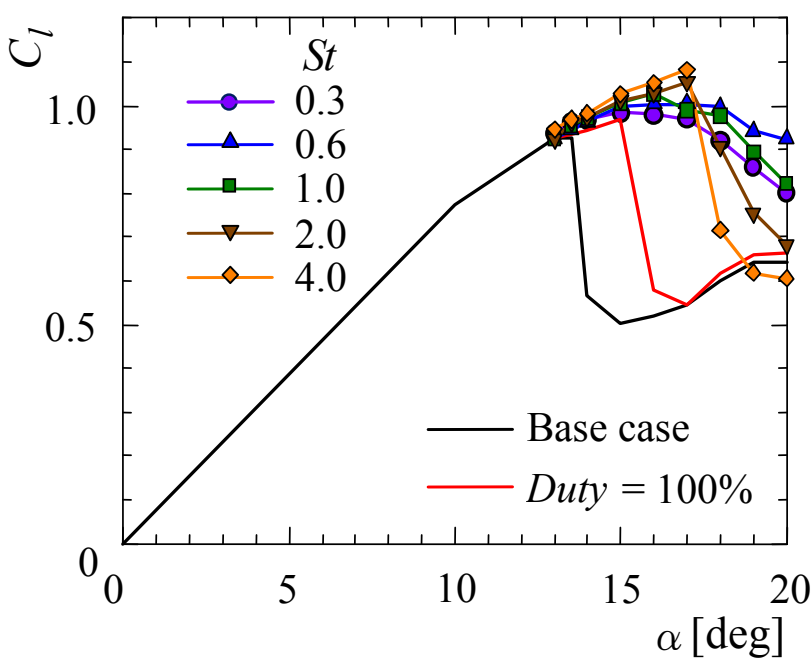

Fig. 4 Lift coefficients for Base case — and Duty $=100 \%$ - versus angle of attack. The effects of pulse modulation $(S t=0.3,0.6,1.0,2.0$ and 4.0$)$ for Duty $=10 \%$ also shown. The application of pulse modulation is able to delay a stall much longer than for either the Base case or for Duty $=100 \%$. It can clearly be seen that $C_{l}$ falls sharply after reaching a maximum $(S t=4.0)$, while for $S t=0.6$, the value of $C_{l}$ falls gradually. 
Generally, the ratio of $C_{l} / C_{d}$ increases with the angle of attack until the airfoil stalls. Therefore, we can say that before the airfoil stalls, $S t=4.0$, for which the value of $C_{l}$ is the highest (Fig. 4), will generally have the lowest $C_{d}($ Fig. 5). However, it is clearly different in the case of a high angle of attack ( $\alpha=18^{\circ}$ to $20^{\circ}$ ), where both the drag and lift coefficients are highest when $S t=0.6$. This phenomenon may occur as a result of the rapid change in the flow field caused by the pulse modulation. (This rapid change in the flow field is shown later in Fig. 16, where the fluctuating velocity is higher when $S t=0.6$ rather than when $S t=4.0$ ). This may increase the lift force and also create a frictional force on the wing surface that causes an increase in the drag coefficient.

In the stall control condition, most of the pulse modulation cases do not affect the $C_{l}$ value, except for Duty $=80 \%$. This is shown in Fig. 6, where Duty $=80 \%$ shows an optimum value of $C_{l}$ at $S t=0.6$, but this $C_{l}$ value is much lower than that for other values of Duty.

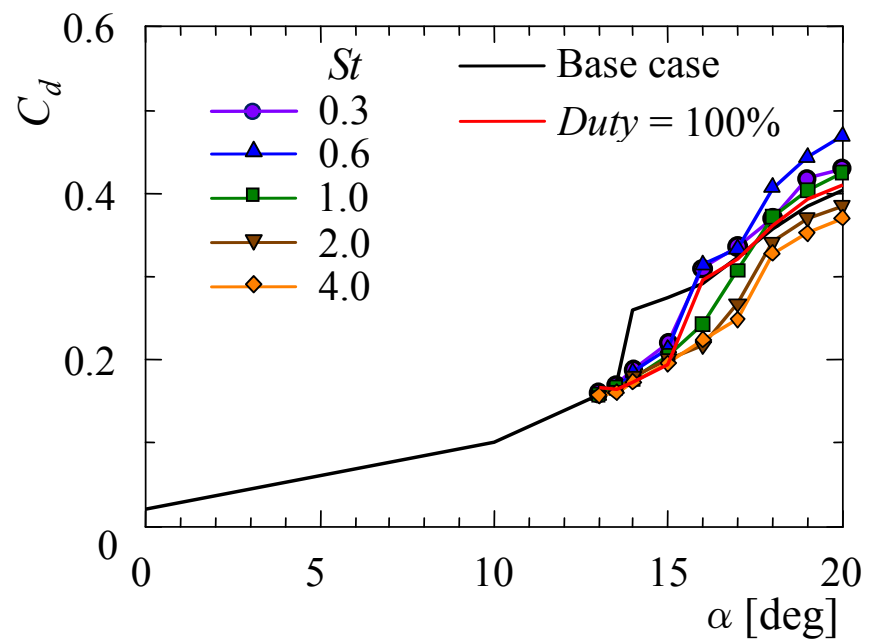

Fig. 5 Drag coefficients for Base case — and Duty $=100 \%-$ for different angles of attack. The effect of pulsemodulation $(S t=0.3,0.6,1.0,2.0$ and 4.0$)$ for Duty $=10 \%$ are also shown.

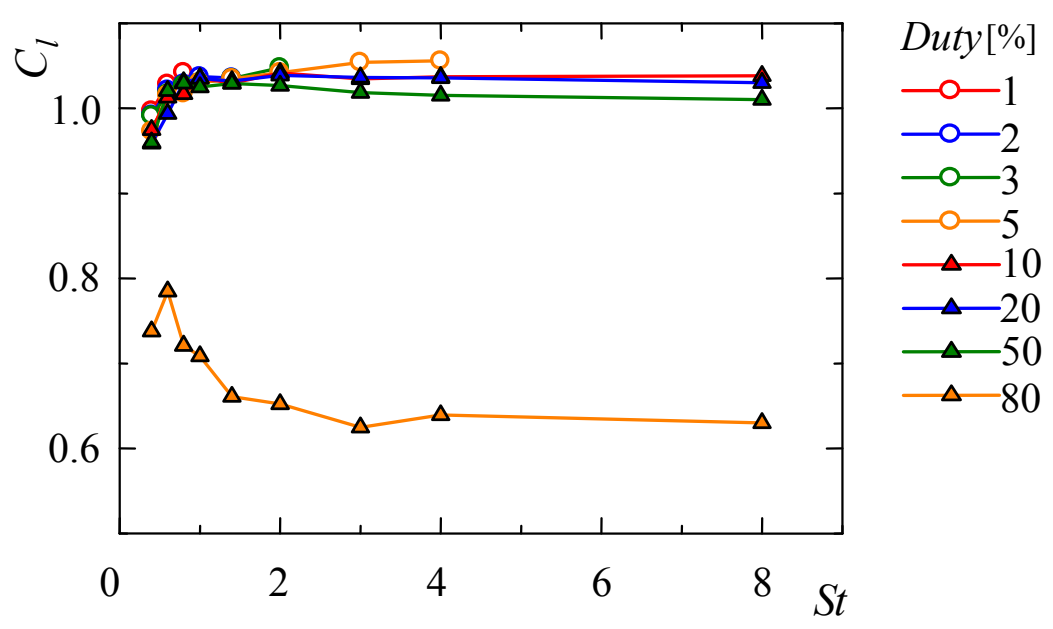

Fig. 6 Effect of modulation frequency on $C_{l}$ when $\alpha=16^{\circ}$ (stall control condition). Most of the pulse modulation settings do not affect the $C_{l}$ value except for Duty $=80 \%$. For Duty $=80 \%$, an optimum value of $C_{l}$ is obtained when $S t=$ 0.6 , however, this $C_{l}$ value is much lower than that for other values of Duty. 
For a high angle of attack, however, most of the duty ratios produce an optimum $C_{l}$ at $S t=0.6$ (Fig. 7). Then, the $C_{l}$ value falls as $S t$ increases. In addition, at Duty $=20 \%, 50 \%$, and $80 \%$, the lift coefficient is constant when $S t$ is greater than 4.0. These findings suggest that the pulse-modulation drive may be able to improve the lift coefficient for a high angle of attack at $S t=0.6$, rather than in the stall control situation. In Figs. 6 and 7, the data is not complete for Duty= $1 \%, 2 \%, 3 \%$ and $5 \%$ because it was not possible to produce a sine wave signal above the upper limit of St.

Figure 8 shows the effect of the duty ratio on the lift coefficient at $\alpha=18^{\circ}$. We found that the lift coefficients are highest when $S t=0.6$, regardless of the duty ratios, while they are lowest when $S t=8.0$. Every value of $S t$ was found to exhibit an optimum lift coefficient at certain duty ratios. Thus, it appears that there is an optimum actuation time for the applied pulse modulation frequency. For example, the optimum lift coefficients for $S t=0.6,1.0,2.0$ and 4.0 are obtained at Duty $=1.5 \%, 2.5 \%, 5 \%$ and $10 \%$ respectively. In other words, the optimum actuation time for the high angle of attack is about $0.25 \mathrm{~ms}$.

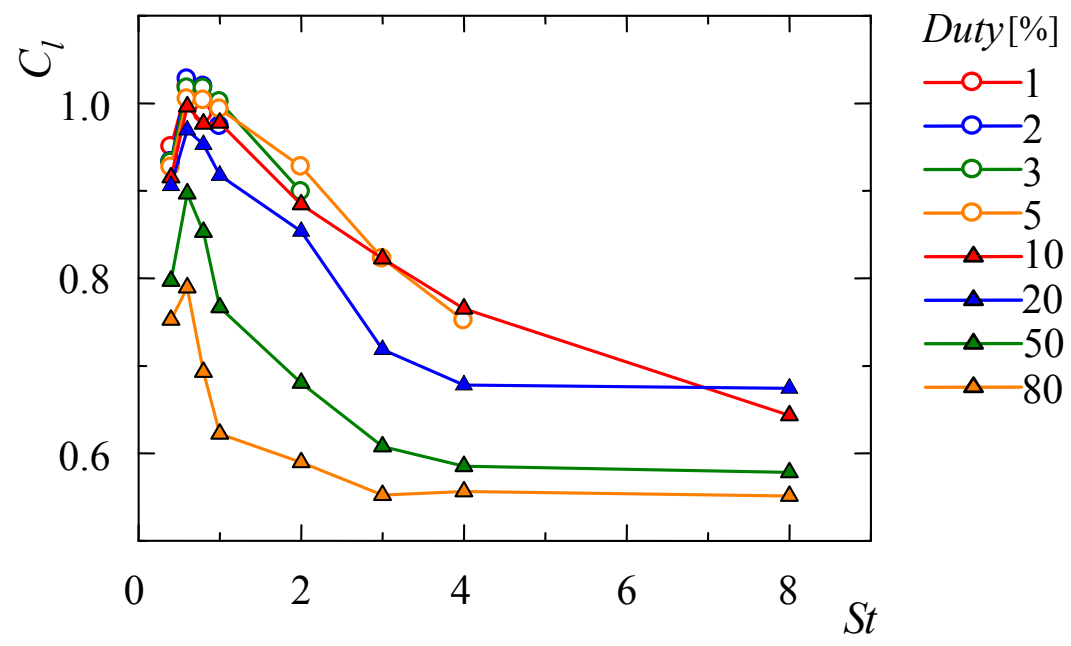

Fig. 7 Effect of modulation frequency on $C_{l}$ when $\alpha=18^{\circ}$ (high angle of attack). Most of the pulse modulations produce an optimum value of $C_{l}$ when $S t=0.6$. This means that pulse modulation is able to affect $C_{l}$ at a high angle of attack. In addition, for Duty $=20 \%, 50 \%$, and $80 \%$, the value of the lift coefficient is constant when $S t$ is greater than 4.0.

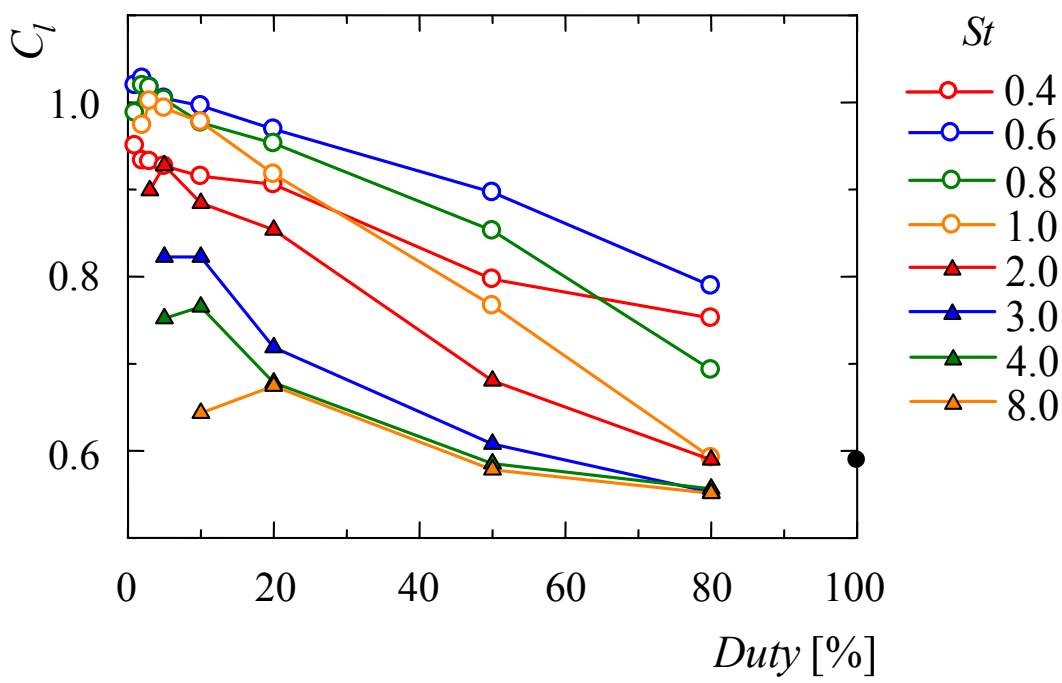

- Duty $100 \%$

Duty $[\%]$

Fig. 8 Effect of duty ratio on lift coefficient at $\alpha=18^{\circ}$. When $S t=0.6$, the lift coefficients are highest for all Duty values, while $S t=8.0$ produces the lowest lift coefficients. Furthermore, every $S t$ value produces an optimum lift coefficient at certain duty ratios. Thus, there appears to be an optimum actuation time depending on the applied pulse modulation frequency. 


\subsection{Flow around the airfoil}

We performed hot wire velocity measurements, the results of which are shown in Figs. 9 and 10. Figure 9 shows the stall control case, in which the mean velocites near the airfoil surface when $S t=0.6$ and 4.0 are higher than in the base case. Therefore, we can expect that separation will not occur when $S t=0.6$ and 4.0. Figure 10 shows the high angle of attack case, for which we can predict that separation will occur when $S t=4.0$. This is because the mean velocity above the airfoil is as low as in the base case. When $S t=0.6$, the mean velocity appears to be higher above the airfoil, indicating that separation has not occurred. To confirm this, however, an analysis of the fluctuating velocity and captured images should be implemented.

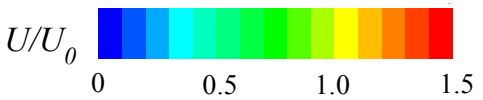

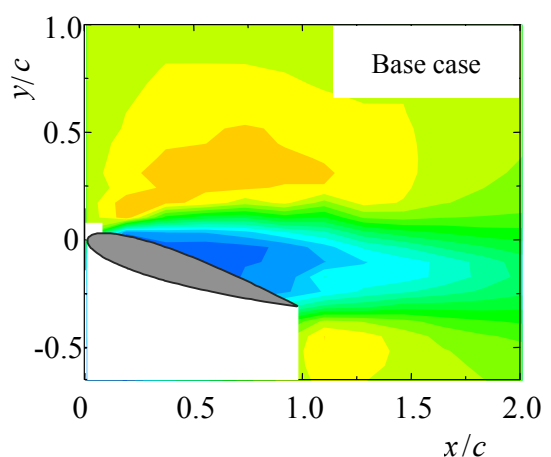
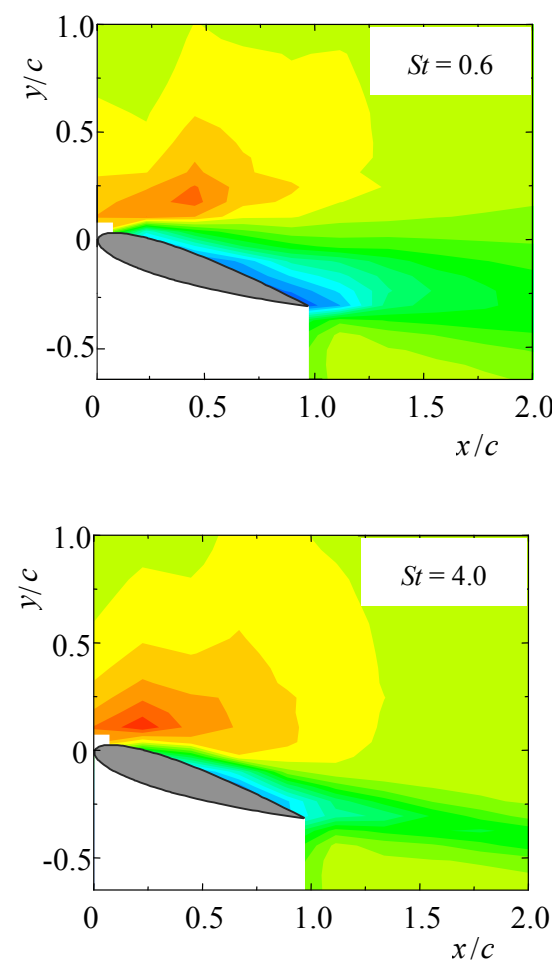

Fig. 9 Time-mean velocity contour map at $\alpha=16^{\circ}$. The mean velocity near the airfoil surface when $S t=$ 0.6 and 4.0 is higher compared to that in the base case. Therefore, separation may not occur when $S t$ $=0.6$ and 4.0 .
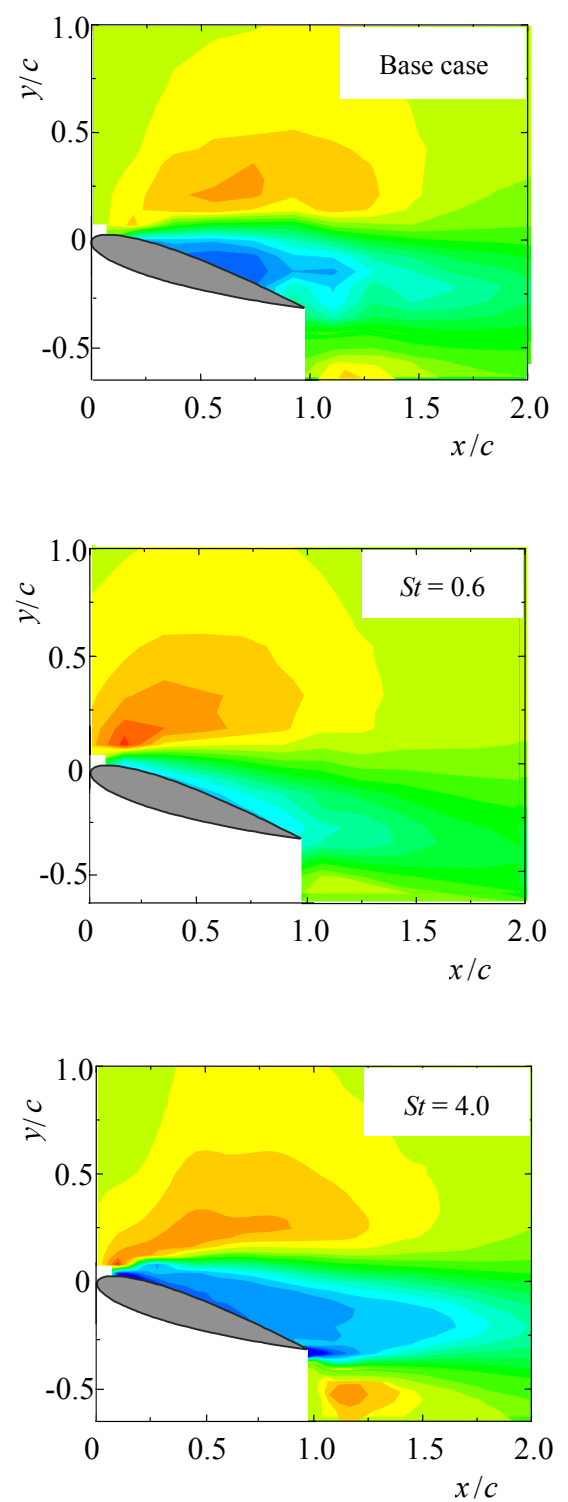

Fig.10 Time-mean velocity contour map at $\alpha=18^{\circ}$. Separation may occur when $S t=4.0$ because the mean velocity above the airfoil is as low as in the base case. When $S t=0.6$ case, it is predicted separation has not occurred due to the higher mean velocity above airfoil. 
Figure 11 and 12 show the turbulence intensity contour maps. In the stall control case (Fig.11), the turbulence intensities for $S t=0.6$ are much higher than in the base case. The turbulence intensity near the airfoil surface is quite small when $S t=4.0$. This agrees well with the findings shown in Fig. 9 (no separation when $S t=4.0$ ). In the base case, a moderate level of velocity fluctuation occurs in the vicinity of the trailing edge. When $S t=0.6$, however, a high level of turbulence is observed along the entire wing surface and separation probably occurs.

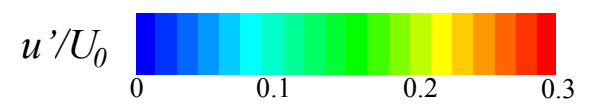

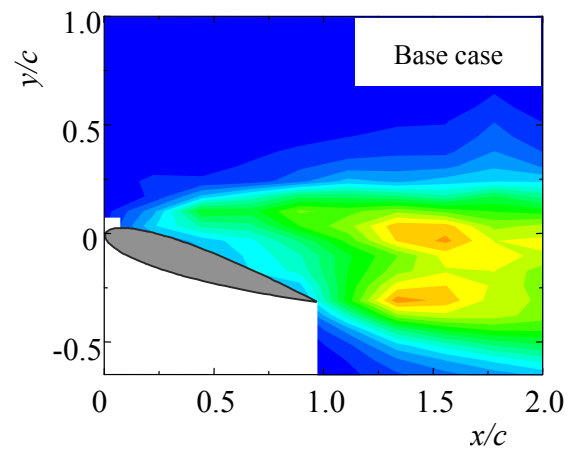
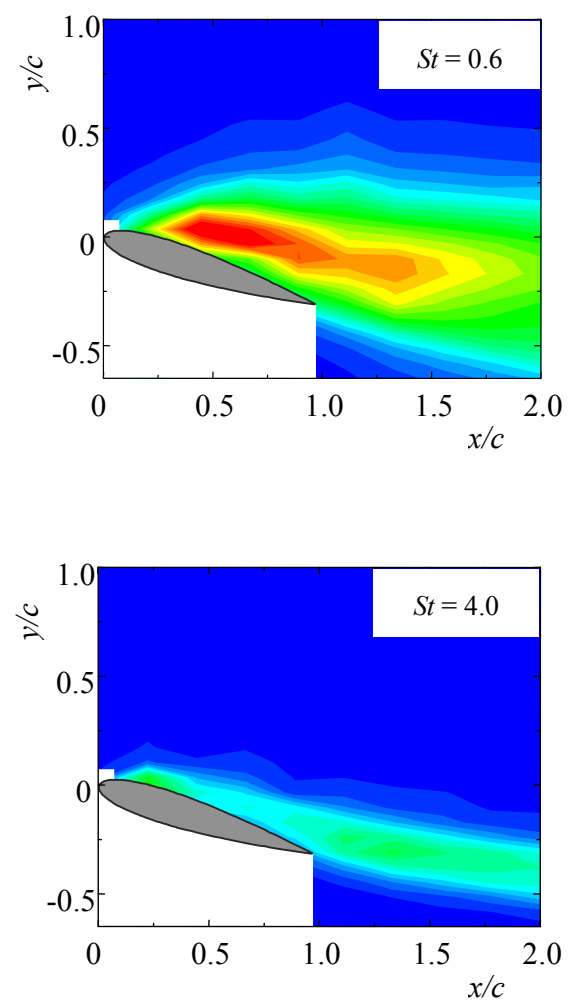

Fig. 11 Turbulence intensity contour map at $\alpha=16^{\circ}$. The turbulence intensities when $S t=0.6$ are much higher than in the base case while the turbulence intensity is quite small near the airfoil surface when $S t=4.0$.
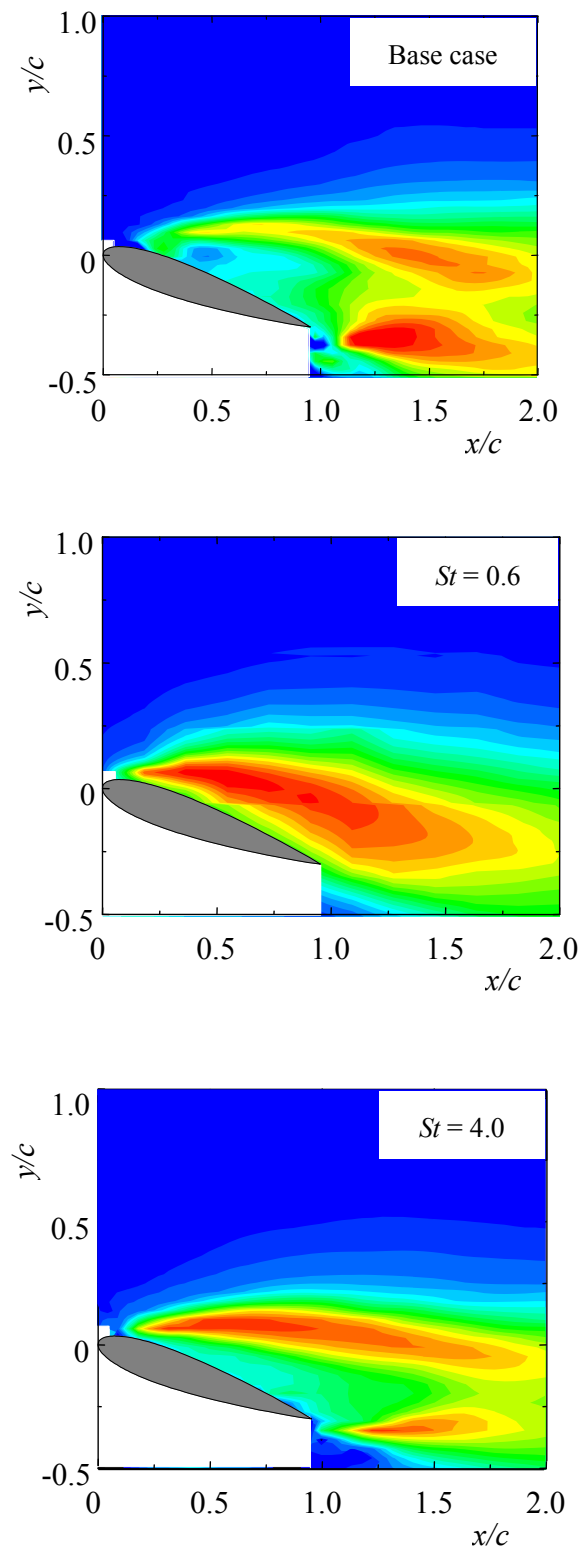

Fig.12 Turbulence intensity contour map at $\alpha=18^{\circ}$. At the high angle of attack, the turbulence intensities increase around the airfoil in every cases (Base case, $S t=0.6$ and $S t=4.0)$. 
For the high angle of attack (Fig. 12), the turbulence intensity increases around the airfoil in every cases (Base case, $S t=0.6$ and $S t=4.0)$. A high level of turbulence is detected with the fluctuating velocity across the entire airfoil surface $(S t=0.6)$. This suggests that unsteady separation may be occurring, which does not agree with the result shown in Fig. 10 for $S t=0.6$. In other words, the result shown in Fig. $12(S t=0.6)$ does not support the result shown in Fig. 10 $(S t=0.6)$. This should be clarified by visualization. On the other hand, when $S t=4.0$, the intensity of the turbulence is high, mainly near the leading edge and also near the wake region. This corresponds to the result shown in Fig. 10 (St= 4.0) where we predicted that the flow would separate above the airfoil. For the base case, the turbulence intensity is much higher near the trailing edge. Therefore, we can conclude that the turbulence intensity varies with the value of $S t$.

Images captured using the smoke method for both the stall control and high angle of attack conditions are shown in Figs. 13 and 14, respectively. Here, $T$ is the period of the ON-OFF cycle. From Fig. 13, we can see that $S t=4.0$ produces a better flow attachment than $S t=0.6$. We can also see that a continuous small-scale vortex is produced from the leading edge, with the airflow attaching to the full wing surface for the entire period, $T$. For $S t=0.6$, however, a large-scale vortical flow slowly develops from $1 / 3 T$ until it covers the entire airfoil surface at $3 / 3 T$. From this we can say that separation occurs in some period of time for $S t=0.6$. Thus, we can conclude that raising the value of $S t$ is an effective means of increasing the airfoil performance in the stall control condition.
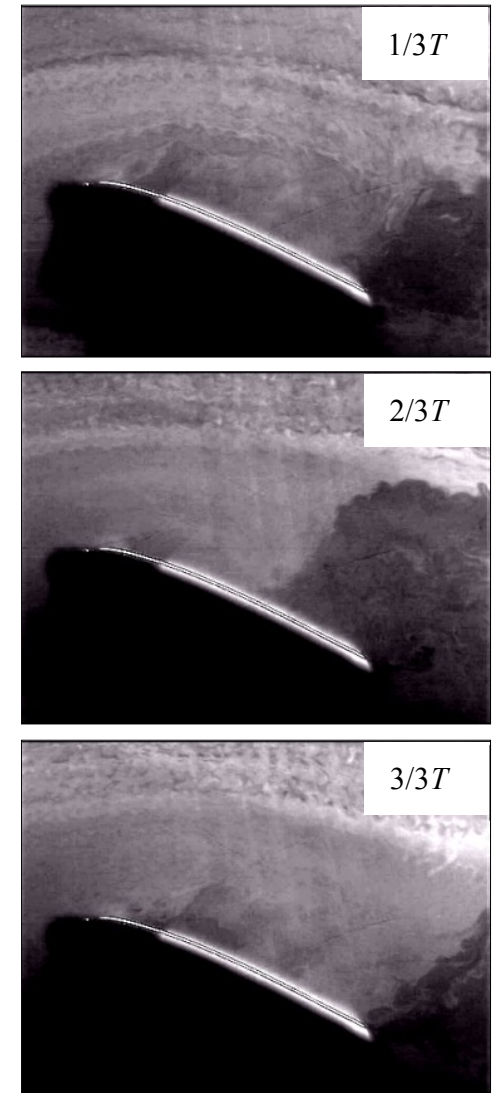

(a) $S t=0.6$
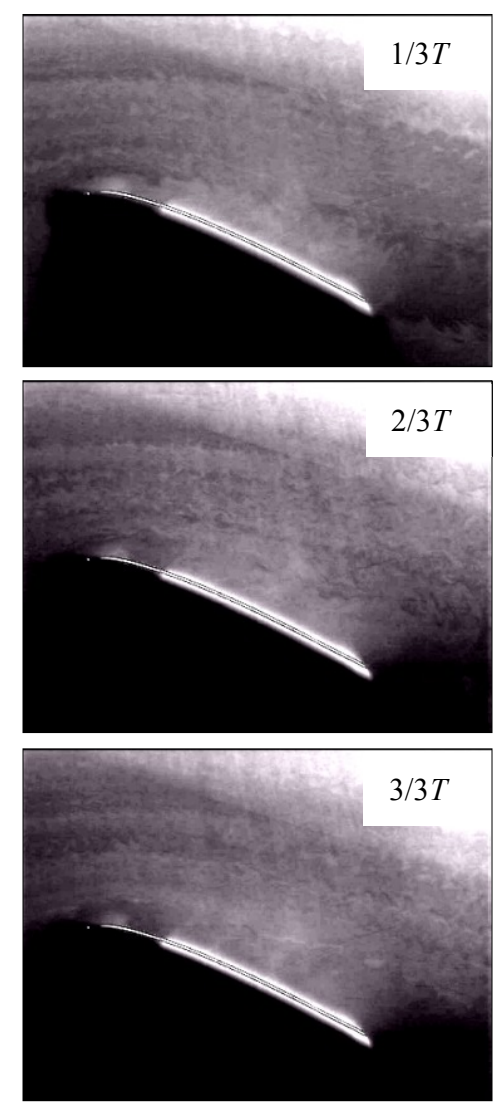

(b) $S t=4.0$

Fig.13 Captured image of airflow over an airfoil for (a) $S t=0.6$ and (b) $S t=4.0$ at $\alpha=16^{\circ}$ during period $T$. Here, $T$ is the period of the ON-OFF cycle. $S t=4.0$ shows a better flow attachment than when $S t=0.6$. Furthermore, a continuous small-scale vortex can been seen being produced from the leading edge, with the airflow attaching to the entire wing surface throughout the period, $T$. When $S t=0.6$, a large-scale vortical flow slowly develops from $1 / 3 T$ until it covers the entire airfoil surface at $3 / 3 T$. 
Figure 14(a) shows how the separated shear layer rolls up near the leading edge when $S t=0.6$. As a result, the vortex structure grows and flows downstream near the wing surface. It is interesting that this flow behavior is very similar to that of the stall control condition (Fig. 13(a)). This is because the velocity above the airfoil decreases with an increase in the angle of attack. A long period of pulse modulation $(S t=0.6)$ may cause the separated shear layer near the leading edge to roll up and develop. These results suggest that the pulse-modulation drive is able to manipulate the flow behavior above the airfoil in both the high angle of attack and stall control conditions. When $S t=4.0$ (Fig. 14(b)), however, the separation seems to be larger than when $S t=0.6$. The flow attachment area is limited near the leading edge of the airfoil. In this case, a short pulse modulation period $(S t=4.0)$ produces a small-scale vortex that separates from the wing surface.
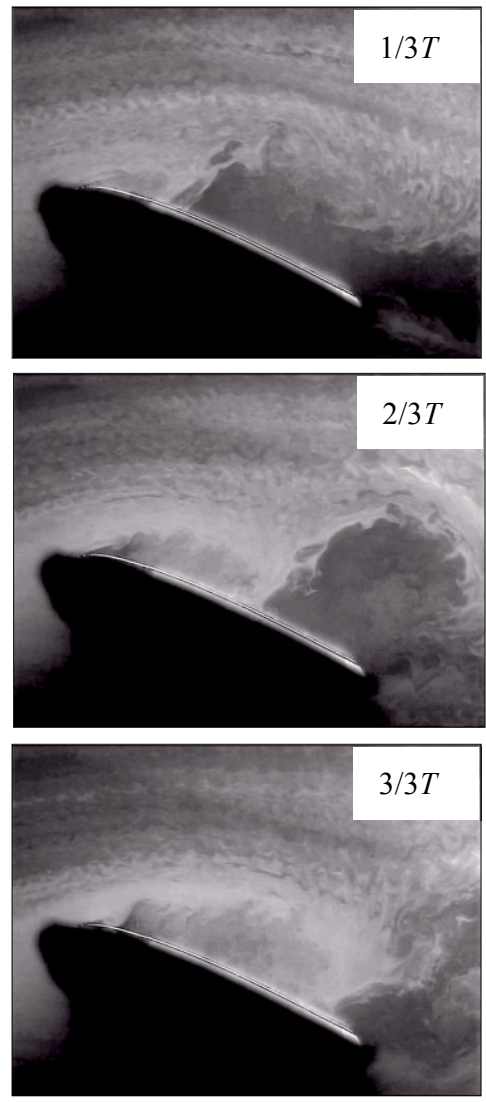

(a) $S t=0.6$
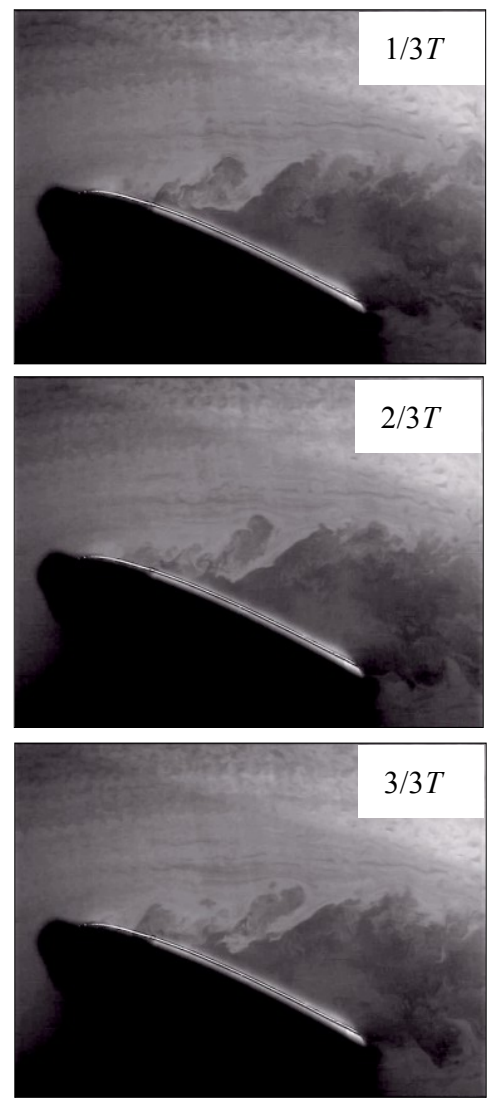

(b) $S t=4.0$

Fig.14 Captured image of airflow over an airfoil for (a) $S t=0.6$ and (b) $S t=4.0$ at $\alpha=18^{\circ}$ during period $T$. Here, $T$ is the period of the ON-OFF cycle. When $S t=0.6$, the separated shear layer rolls up near the leading edge. When $S t=$ 4.0 , however, the separation appears to be larger than that when $S t=0.6$. The flow attachment area is limited to the region near the leading edge of the airfoil. In this case, a short-period pulse modulation $(S t=4.0)$ produces a small-scale vortex that separates from wing surface. 


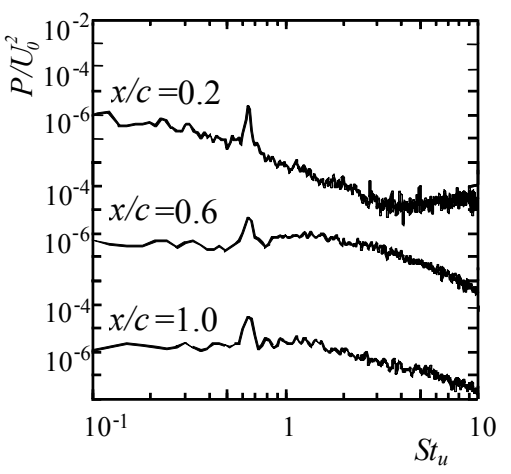

Base case

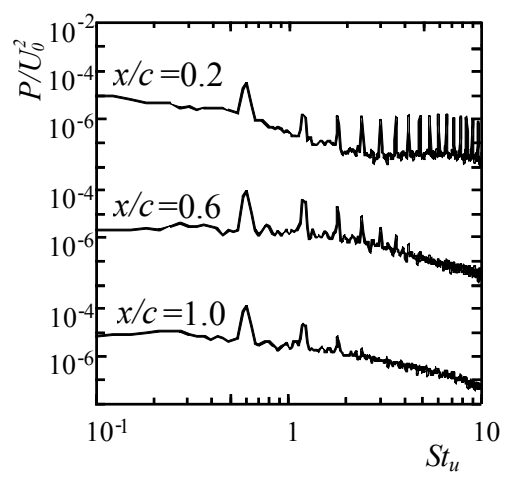

$\underline{S t=0.6}$

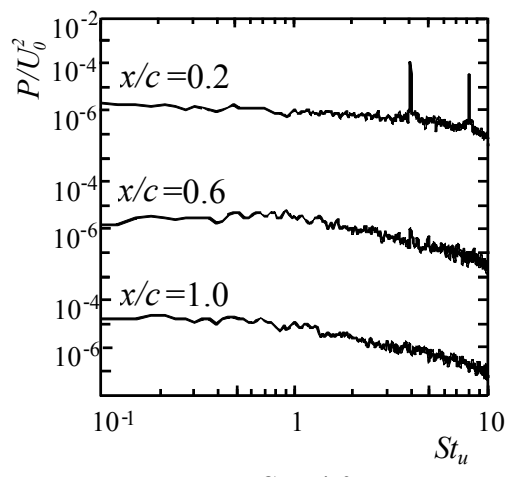

$\underline{S t=4.0}$

Fig.15 Power spectra of fluctuating velocity at the outer edge of the shear layer region for the base case, $S t=0.6$ and $S t=$ 4.0. These show the energy of the fluctuating velocity when position $x / c=0.2,0.6$ and 1.0. For the base case, there is a dominant frequency when $S t=0.64$, which corresponds to natural vortex shedding. When the actuator is driven at $S t=0.6$, the corresponding frequency fluctuation is detected at all positions $(x / c=0.2,0.6$, and 1.0$)$. The energy of the fluctuating velocity is detected only near the leading edge $(x / c=0.2)$ when $S t=4.0$.

Figure 12 shows how the turbulence intensities vary depending on the value of $S t$. To determine the reason for this, we performed a frequency analysis of the fluctuating velocity. Figure 15 shows the power spectra for the fluctuating velocity at the outer edge of the shear layer region. For the base case, there is a dominant frequency at $S t=0.64$ that corresponds to natural vortex shedding. When the actuator is driven at $S t=0.6$, the corresponding frequency velocity fluctuation is detected at all positions $(x / c=0.2,0.6$, and 1.0). This result agrees with that shown in Fig. $12(S t=0.6)$ where the fluctuating velocity covers almost the entire airfoil surface. For the same case, if the actuator is driven at its natural frequency of 0.64 , there will be no difference in the flow behavior. The corresponding frequency will be detected for every position. Around this frequency, it is thought that the lock-in phenomenon will occur in the same way as in the case of a circular cylinder. On the other hand, the fluctuating velocity energy is detected only near the leading edge $(x / c=0.2)$ when $S t=4.0$. This agrees with the result shown in Fig. $12(S t=4.0)$ where the fluctuating velocity is detected mainly near the leading edge. Further analysis of the fluctuating velocities will be necessary to confirm this.

Figure 16 shows a contour map of the dominant frequency components of the fluctuating velocity for the base case, $S t=0.6$, and $S t=4.0$. It shows that the energy levels of the fluctuating velocities are higher above the midpoint of the airfoil at $S t=0.6$. Comparing this with the images of the flow, this corresponds to vortex shedding at the leading edge, growing to a large vortex flowing downstream along the airfoil surface. For $S t=4.0$, however, the region in which there is a high level of fluctuating velocities is limited to near the leading edge. A small-scale vortex is formed near the leading edge, after which it diverges away from the wing surface. Then, the vortex structures appear to disperse slowly downstream. In the base case, there is high-turbulence fluctuation in the vicinity of the trailing edge. Figure 16 shows why the value of $C_{l}(S t=4.0)$ falls rapidly compared to when $S t=0.6$, where $C_{l}$ falls gradually from $\alpha=17^{\circ}$ to $\alpha=18^{\circ}$ (Fig. 4). Lastly, we can say that these results suggest that vortex shedding can be manipulated by the application of a pulse- modulation frequency so as to attain better flow attachment. 


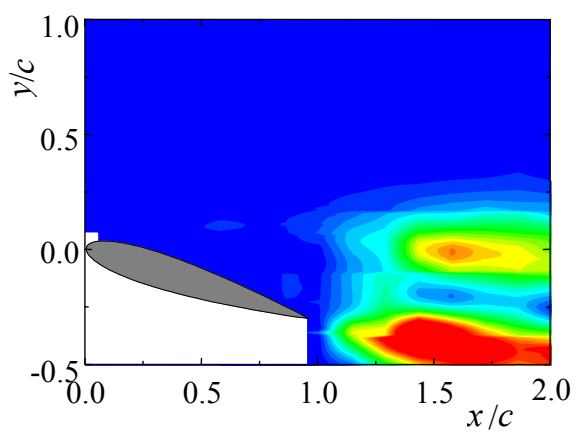

$\underline{\text { Base case }}$

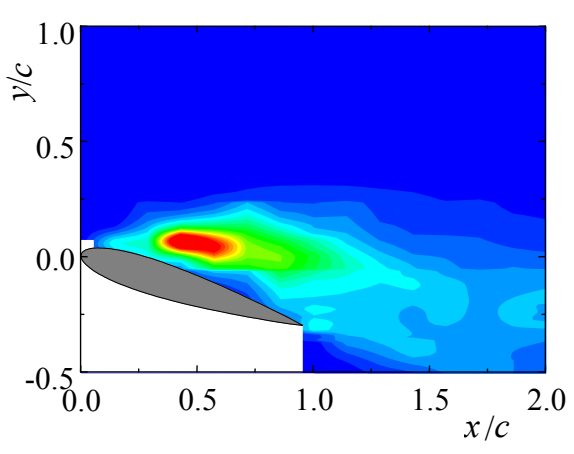

$\underline{S t=0.6}$
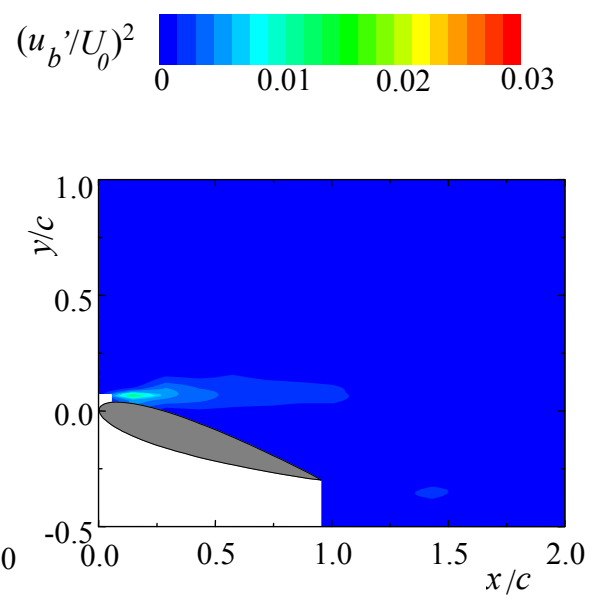

$\underline{S t=4.0}$

Fig.16 Contour map of dominant frequency components of fluctuating velocity at $\alpha=18^{\circ}$ for the base case, $S t=0.6$ and $S t$ $=4.0$. The energy levels of the fluctuating velocities are higher at the midpoint above airfoil at $S t=0.6$. When $S t=$ 4.0, however, the region where there is a high level of fluctuating velocities is limited to near the leading edge. A small-scale vortex is formed near the leading edge and then diverges away from the wing surface. For the base case, a high level of turbulence fluctuation exists in the vicinity of the trailing edge.

\section{Conclusion}

We performed an experimental study of a means of controlling flow separation on an airfoil by using a plasma actuator. The results can be summarized as follows:

1. Even though Duty $=100 \%$ causes a stall to occur at $\alpha=15^{\circ}$, the application of ON-OFF control is able to maintain the increment of the $C_{l}$ value. Furthermore, when $S t=4.0$, the lift coefficient is reduced sharply after reaching a maximum, while when $S t=0.6$, it falls gradually. For a high angle of attack $\left(\alpha=18^{\circ}\right)$ when the angle of attack exceeds the maximum $C_{l}$, there is an optimum pulse length for effectively controlling the flow.

2. Pulse-modulated drive is able to manipulate the behavior of the flow above the airfoil in the high angle of attack. When $S t=0.6$, a large vortex appears and covers almost the entire airfoil surface. When $S t=4.0$, however, a smallscale vortex structure forms near the leading edge and then diverges away from the wing surface.

3. Setting $S t=4.0$ is an effective means of increasing the airfoil performance for the stall control condition $\left(\alpha=16^{\circ}\right)$. For the high angle of attack case, however, we found that setting $S t=0.6$ increases the lift coefficient of the airfoil. 


\section{References}

Amitay, M. and Glezer, A., Role of actuation frequency in controlled flow reattachment over a stalled airfoil, AIAA Journal Vol. 40, No. 2 (2002), pp.209-216.

Asada, K., Ninomiya, Y., Oyama, A. and Fujii, K., Airfoil flow experiment on the duty cycle of DBD plasma actuator, AIAA paper 2009-531, $47^{\text {th }}$ AIAA Aerospace Sciences Meeting (2009).

Balcon, N., Benard, N. and Moreau, E., Formation process of the electric wind produced by a plasma actuator, IEEE Transactions on Dielectric and Electrical Insulation,Vol. 16, No. 2 (2009), pp.463-469.

Bernard, N., Bonnet, J.P., Moreau, E., Griffin, J. and Cattafesta, L.N., Benefits of using hysteresis effects for closed-loop separation control by plasma actuator, AIAA paper 2010-4259 (2010).

Goksel, B., Greenblatt, D., Rechenberg, I., Nayeri, C. N. and Paschereit, C. O., Steady and unsteady plasma wall jets for separation and circulation control, AIAA paper 2006-3686, $3^{\text {rd }}$ AIAA Flow Control Conference (2006).

Greenblat, D., Schulman, M. and Ben-Harav, A., Vertical axis wind turbine performance enhancement using plasma actuators, Renewable Energy, Vol. 37 (2012), pp.345-354.

Greenblatt, D., Goskel, B., Rechenberg, I., Schule, C.Y., Romann, D. and Paschereit, C.O., Dielectric barrier discharge flow control at very low flight Reynolds numbers, AIAA Journal Vol. 46, No. 6 (2008).

Hasebe, H., Naka, Y. and Fukagata, K., An attempt for suppression of wing-tip vortex using plasma actuators, Journal of Fluid Science and Technology, Vol. 6, No. 6 (2011), pp.976-988.

Jolibois, J., Forte, M. and Moreau, E., Application of an AC barrier discharge actuator to control airflow separation above a NACA 0015 airfoil: Optimization of the actuation location along the chord, Journal of Electrostatics, Vol.66 (2008), pp.496-503.

Jukes, T.N. and Choi, K-S., Flow control around a circular cylinder using pulsed dielectric barrier discharge surface plasma, Physics of Fluids, Vol. 21, (2009) 084103.

Post, M.L. and Corke, T., Separation control on high angle of attack airfoil using plasma actuators, AIAA Journal Vol. 42, No. 11 (2004), pp.2177-2178.

Rethmel, C., Little, J., Takashima, K., Sinha, A., Adamovich, I. and Samimy, M., Flow separation control over an airfoil with nanosecond pulse driven DBD plasma actuators, AIAA paper 2011-487, $49^{\text {th }}$ AIAA Aerospace Sciences Meeting (2011).

Sidorenko, A., Zanin, B., Postnikov, B., Budovsky, A., Starikovskii, A., Roupassov, D., Zavialov, I., Malmulth, N., Smereczniak, P. and Silkey, J., Pulsed discharge actuators for rectangular wings separation control, AIAA paper 2007-941, 45 ${ }^{\text {th }}$ AIAA Aerospace Sciences Meeting and Exhibit (2007), pp.11422-11432.

Sosa, R. and Artana, G., Steady control of laminar separation over airfoils with plasma sheet actuators, Journal of Electrostatics, Vol. 64 (2006), pp.604-610.

Yang, Z., Haan, F.L., Hui, H. and Ma, H., An experimental investigation on the flow separation on a low-Reynolds-number airfoil, AIAA paper 2007-0275, 45 ${ }^{\text {th }}$ AIAA Aerospace Sciences Meeting and Exhibit (2007). 